\title{
Simultaneous Multichannel Time Domain Equalizer Design Based On The Maximum Composite Shortening SNR
}

\author{
Milos Milosevic*, Lucio F. C. Pessoa**, and Brian L. Evans* 1 \\ *Dept. of Electrical and Computer Eng., The University of Texas, Austin, TX 78712 \\ **7700 West Parmer Lane MD: TX32-PL30, Motorola, Inc., Austin, TX 78729
}

\begin{abstract}
A time domain equalizer (TEQ) is a finite impulse response filter that shortens the channel impulse response (CIR) to mitigate inter-symbol interference (ISI). Melsa, Younce, and Rohrs minimized ISI in the time domain by maximizing the shortening signal-tonoise ratio (SSNR) of the energy in a target window to the energy outside the target window in the shortened CIR. Infinite SSNR means zero ISI. Melsa, Younce, and Rohrs also developed a joint channel shortening method to design a single TEQ to shorten a channel and a near-end echo impulse response. In this paper, we extend the joint SSNR method to design a single TEQ to shorten multiple channels by maximizing the composite SSNR. The composite SSNR is a weighted sum of normalized channel SSNRs. The normalized SSNR is the ratio of the energy in the target window samples to the energy of all samples in the shortened CIR and has a range of $[0,1]$ so that it is better suited for numerical stability and fixed-point implementation. Our proposed method outperforms the joint channel shortening method. because it achieves higher weighted sum of SSNRs of the used channels.
\end{abstract}

\section{Introduction}

Discrete multi-tone (DMT) is a multicarrier modulation (MCM) method in which the available bandwidth of a communication channel, such as twisted-pair copper media, is divided into numerous subchannels or bins via a fast Fourier transform (FFT). Modulation by the inverse fast Fourier transform (IFFT) and demodulation by FFT create nearly orthogonal subchannels.

DMT has been standardized in $[1,2,3,4]$. A similar MCM that has been adopted in the Digital Video

\footnotetext{
${ }^{1}$ B. Evans was funded by The State of Texas Advanced Technology Program under project 0036580614-2001. E-mail: Lucio.Pessoa@motorola.com, and \{milos, bevans $\}$ @ece.utexas.edu.
}

Broadcasting (DVB) [5] and HYPERLAN-2 [6] standards is called Orthogonal Frequency Division Multiplexing (OFDM).

A guard period called the cyclic prefix (a copy of the last $\nu$ samples of the DMT symbol) is inserted between DMT symbols to reduce inter-carrier interference (ICI) and inter-symbol interference (ISI). A channel impulse response (CIR) longer than $\nu+1$ samples causes both ICI and ISI. In ADSL, $\frac{\nu}{N}=\frac{1}{16}$, where $N$ is the DMT symbol length. For downstream transmission, $N=512$ and $\nu=32$. For the upstream, $N=64$ and $\nu=4$.

Time domain equalization eliminates the ISI and ICI from the received DMT signal by shortening the channel impulse response. In a traditional discrete multitone equalizer, the time domain equalization is implemented by a single finite impulse response (FIR) filter, called the time domain equalizer (TEQ).

For DMT modulation, many methods exist $[7,8,9$, $10,11]$ for the design of a TEQ for the single-channel case. A few deal with the issues of designing a TEQ for multiple channels. Melsa, Younce, and Rohrs [10] propose to minimize ISI by maximizing the shortening signal-to-noise ratio (SSNR). The SSNR is the ratio of the energy present in the target shortened impulse response (SIR) window of length $\nu+1$ samples to the energy outside of the window. The MSSNR method is based on an observation that reducing the energy of the SIR outside the target window will reduce ISI and ICI [12]. The authors in [10] also propose a method for jointly shortening two channels, which we refer to as Joint MSSNR. This method jointly shortens a single CIR and the near-end echo impulse response using a single TEQ FIR, thus reducing ISI and simplifying the removal of near-end echo.

Farhang-Boroujeny and Ding [13] propose a DMT TEQ design procedure for a large number of transmission channels. They identify 20 classes of channels through CSA loop simulation and measurement in the Singapore CSA. The modem measures the transmission channel impulse response during the initialization se- 
quence. It then uses the measured impulse response to find the best match among the magnitude responses of the representative channel class members. The classification process retrieves the matching target impulse response and the transmission delay. The target impulse response and transmission delay for each class is saved off-line as a by-product of MMSE TEQ design for the representative members of each class. After classification, the TEQ coefficients are obtained online by an MMSE design method that uses the retrieved target impulse response and the transmission delay.

G.992.2 Fast Retrain standard [3] enables an ADSL modem to establish the connection quickly after the link has been severed due to a disturbance on the line, e.g. phone ringing on the same line. The modem memorizes the link environment, e.g. the TEQ. in several different time instances. After the disturbance has passed and the G.992.2 Fast Retrain initialization sequence is in progress, the modem recognizes the new channel (in a way not defined by the standard) and retrieves the appropriate TEQ among other parameters. The method of selection of the best TEQ for the new channel is not defined in the standard.

A TEQ design procedure whose result is a TEQ that shortens multiple channels would simplify the procedure envisioned under the Fast Retrain standard or could reduce the number of different CSA classes of channels as in [13].

In this paper, we propose the Maximum Composite SSNR (MCSSNR) method to design a single FIR TEQ to shorten more than one channel by maximizing a sum of modified channel SSNRs called CSSNR. The modified SSNR measure that we propose uses the ratio of the channel energy inside the target window to the overall energy. The modified SSNR measure takes values from 0 (maximum ISI) to 1 (minimum ISI), inclusive, and is conveniently represented on fixed-point programmable digital signal processors used in commercial ADSL modems. Our proposed method outperforms the joint channel shortening method of Melsa, Younce, and Rohrs (Joint MSSNR) because it achieves higher CSSNR of the used channels and data rate/channel. Our results show that a successful SSNR-based TEQ design does not necessarily maximize the bit rate of a DMT system. The contributions of this paper are: (1) a modified SSNR ratio suitable for the fixed-point implementation, and (2) a multichannel TEQ design algorithm.

\section{Composite SSNR Objective Measure}

We consider a discretized transmission channel modelled as an FIR filter with an impulse response of $N$ coefficients denoted $\mathbf{h}$, and an $M$-tap long FIR TEQ denoted $\mathbf{w}$. The cyclic prefix is $\nu$ samples long. The linear convolution of the CIR and TEQ results in an effective (and hopefully shortened) channel $\mathbf{h}_{\text {eff }}$.

Following [10] we partition $\mathbf{h}_{\text {eff }}$ into two channels: (1) $\mathbf{h}_{\text {win }}$, which consists of samples of $\mathbf{h}_{\text {eff }}$ lying within the desired $\nu+1$ window, and (2) $\mathbf{h}_{\text {wall }}$, which consists of the remaining samples of $\mathbf{h}_{\mathrm{eff}}$. The effective CIR $\mathbf{h}_{\text {eff }}$ can be written as $\mathbf{h}_{\text {eff }}=\mathbf{H w}$ where $\mathbf{H}$ is the $(N+$ $M-1) \times M$ convolution matrix of $\mathbf{h}$. So, $\mathbf{h}_{\mathrm{win}}=\mathbf{H}_{\mathrm{win}} \mathbf{w}$ and $\mathbf{h}_{\text {wall }}=\mathbf{H}_{\text {wall }} \mathbf{w}$ where $\mathbf{H}_{\text {win }}$ consists of $\nu$ rows of $\mathbf{H}$ starting from position $\Delta$, where $\Delta$ is the transmission delay, and $\mathbf{H}_{\text {wall }}$ consists of the remaining rows of $\mathbf{H}$.

We extend the approach to $K$ channels and propose a new composite SSNR measure

$$
p(\mathbf{w})=\sum_{k=1}^{K} \beta_{k} p_{k}(\mathbf{w})=\sum_{k=1}^{K} \beta_{k} \frac{\mathbf{w}^{\mathrm{T}} \mathbf{A}_{k} \mathbf{w}}{\mathbf{w}^{\mathrm{T}} \mathbf{B}_{k} \mathbf{w}}
$$

where $\mathbf{A}=\mathbf{H}_{k \text {,win }}^{\mathrm{T}} \mathbf{H}_{k \text {, win }}, \mathbf{B}_{k}=\mathbf{H}_{k}^{\mathrm{T}} \mathbf{H}_{k}$, and $\beta_{k}$ is the weighting of the contribution of the $k^{\text {th }}$ channel to the overall measure so that $\sum_{k=1}^{K} \beta_{k}=1$ and $\beta>0$.

The ratio $p_{k}(\mathbf{w})$ approaches $\beta_{k}$ as the channel shortening becomes more effective, which is different from the approach in [10] where the value of the ratio can achieve extremely high values leading to potential numerical problems. Our goal is to find $\mathbf{w}_{\text {opt }}$ such that $p(\mathbf{w})$ is maximized.

\section{Maximum Composite SSNR Method}

Maximization of a sum-of-ratios (1) is an active research topic in fractional programming for which a definitive solution has not been discovered $[14,15]$. Thus, we propose a solution that achieves good experimental results, although no guarantee of optimality can be given. An outline of our solution is:

1. find the optimal solutions $\mathbf{w}_{k}^{\text {opt }}$ for $\frac{\mathbf{w}^{\mathrm{T}} \mathbf{A}_{k} \mathbf{w}}{\mathbf{w}^{\mathrm{T}} \mathbf{B}_{k} \mathbf{w}}$ in (1) for each channel $k$,e.g. by constraining $w B_{k} w=1$ and using generalized eigendecomposition to solve $\mathbf{A}_{k} \mathbf{w}=\lambda \mathbf{B}_{k} \mathbf{w}$ (see below)".

2. select the solution $\mathbf{w}=\mathbf{w}_{k}^{\text {opt }}$ for which $p\left(\mathbf{w}_{k}^{\text {opt }}\right)$ in (1) attains the highest value as the initial point, and

3. find the zero of the gradient of $p(\mathbf{w})$, by using the Almogy/Levin iteration [16](discussed below), to find the maximum of the objective function closest to the initial point, a.k.a. the MCSSNR.

A similar approach for a single channel was taken in [11], in which the objective function was the sum of the bit rates per DMT subchannel of a single channel. 
For this solution $p_{k}\left(\mathbf{w}_{k}^{\mathrm{opt}}\right)=\lambda_{k}^{\mathrm{opt}}$. After the solution $\mathbf{w}_{k}^{\text {opt }}$ is found for all $k$, the one $\mathbf{w}_{k}^{\text {opt }}$ for which $p\left(\mathbf{w}_{k}^{\text {opt }}\right)$ has the highest value is chosen for the starting point of an maximization iteration. We apply Almogy and Levin's method [16] to find the root of the gradient of (1). Almogy and Levin intended to maximize a sumof-ratios problem by solving for roots of a parametric problem

$$
b(x)=\max _{x} \sum_{i=1}^{n} \frac{f_{i}(x)}{g_{i}(x)}
$$

which they transformed into the parametric problem

$$
H_{n}(q)=\max _{x} \sum_{i=1}^{n}\left[f_{i}(x)-q_{i} g_{i}(x)\right]
$$

By analogy to the single-ratio maximization (based on Lagrangian multipliers [18]), they defined

$$
q_{i}=\frac{f_{i}\left(x_{p}\right)}{g_{i}\left(x_{p}\right)}
$$

where $x_{p}$ is the solution of the maximization in the previous step. In a single-ratio problem $(n=1)$, the solution is reached at the zero of $H_{1}(q)$ where $q$ is maximized and the point $x$ maximizing the current iteration is the same as $x_{p} . H_{1}(q)$ is a convex, non-increasing function of $q$ with a single root. In that problem, $q$ increases with every iteration, while $H_{1}(q)$ decreases. Analogously, Almogy and Levin solve $H_{n}(q)=0$ to find the optimal solution of the sum-of-ratios problem. Falk and Palocsay [19] show this approach to be erroneous, i.e. in the sum-of-ratios problem, finding a zero of $H_{n}(q)$ does not maximize $b(x)$.

Nonetheless, Almogy and Levin's iteration does efficiently find the roots of the nonlinear function $H_{n}(q)$, which is a convex, non-increasing function of $q$ with a single crossing [14]. Hence, we use their idea, with modifications specific to our problem, to find the optimal root of the gradient of (1) that corresponds to the closest local maximum to the initial point.

The gradient of (1) is

$$
\frac{d p(\mathbf{w})}{d \mathbf{w}}=\sum_{k=1}^{K} r_{k}(\mathbf{w})\left[\mathbf{A}_{k}^{r}-\lambda_{k}(\mathbf{w}) \mathbf{B}_{k}^{r}\right] \mathbf{w}
$$

where

$$
r_{k}(\mathbf{w})=\frac{2 \beta_{k}}{\mathbf{w}^{\mathrm{T}} \mathbf{B}_{k} \mathbf{w}} \text { and } \lambda_{k}(\mathbf{w})=\frac{\mathbf{w}^{\mathrm{T}} \mathbf{A}_{k} \mathbf{w}}{\mathbf{w}^{\mathrm{T}} \mathbf{B}_{k} \mathbf{w}}
$$

Notice that $p(\mathbf{w})=\sum_{k=1}^{K} \lambda_{k}(\mathbf{w})$; thus, increasing $\lambda_{k}(\mathbf{w})$ increases $p(\mathbf{w})$. We write

$$
\mathbf{C}(\mathbf{w})=\sum_{k=1}^{K} \underbrace{r_{k}(\mathbf{w})\left[\mathbf{A}_{k}^{r}-\lambda_{k}(\mathbf{w}) \mathbf{B}_{k}^{r}\right]}_{\mathbf{C}_{k}(\mathbf{w})}
$$

1. Create matrices $\mathbf{A}_{k}$ and $\mathbf{B}_{k}$ for all $k$ in (1).

2. Compute the value of $p(\mathbf{w})$ for impulse $\mathbf{w}$ in (1).

3. Compute the optimal $\mathbf{w}_{k}^{\text {opt }}$ for each channel $\left(\mathbf{w}_{k}^{\text {opt }}\right.$ is the eigenvector of the maximum generalized eigenvalue for the pair of matrices $\mathbf{A}_{k}$ and $\left.\mathbf{B}_{k}[17]\right)$.

4. Compute the value of $\sum_{k=1}^{K} \beta_{k} p_{k}\left(\mathbf{w}_{k}^{\text {opt }}\right)$ - this is the upper bound of the objective function

5. Compute the value of $p\left(\mathbf{w}_{k}^{\text {opt }}\right)$ for each $k$ and chose the maximum of all those values and the associated $\mathbf{w}=\mathbf{w}_{\mathrm{opt}}=\mathbf{w}_{k}^{\mathrm{opt}}$ as the initial point.

6. $r_{k}=\gamma r_{k}+(1-\gamma) \frac{2 \beta_{k}}{\mathbf{w}^{\mathrm{T}} \mathbf{B}_{k} \mathbf{w}}, \forall k$.

7. $\lambda_{k}=\gamma \lambda_{k}+(1-\gamma) \frac{\mathbf{w}^{\mathrm{T}} \mathbf{A}_{k} \mathbf{w}}{\mathbf{w}^{\mathrm{T}} \mathbf{B}_{k} \mathbf{w}}, \forall k$.

8. Compute $\mathbf{C}(\mathbf{w})=\sum_{k=1}^{K} r_{k}\left[\mathbf{A}_{k}^{r}-\lambda_{k} \mathbf{B}_{k}^{r}\right]$.

9. $\mathbf{w}_{\text {new }}=\arg \max \left\{\mathbf{v}^{\mathrm{T}} \mathbf{C}(\mathbf{w}) \mathbf{v},\|\mathbf{v}\|^{2}=1\right\}$.

10. If $\left\|\mathbf{w}_{\text {new }}-\mathbf{w}\right\|_{\infty}<\epsilon$ OR $i>i_{\max }$ return $\mathbf{w}_{\mathrm{opt}}$ and jump to 14 .

11. Calculate $p\left(\mathbf{w}_{\text {new }}\right)$.

12. If $p\left(\mathbf{w}_{\text {new }}\right)<p(\mathbf{w})$ set $\gamma=(1+\gamma) / 2$, ELSE $\mathbf{w}_{\mathrm{opt}}=$ $\mathbf{w}_{\text {new }}$.

13. $\mathbf{w}=\mathbf{w}_{\text {new }} ; i=i+1$; Go back to step 6 and repeat. 14. Done.

Figure 1: Proposed Maximum Composite SSNR TEQ Design algorithm. Initialize the algorithm by setting the iteration counter $i=0$, the smoothing factor $\gamma=0$ and $r_{k}$ and $\lambda_{k}$ to zero for all $k$.

Let

$$
\mathrm{H}(\lambda)=\max _{\mathbf{w},\|\mathbf{w}\|^{2}=1} \mathbf{w}^{\mathrm{T}} \underbrace{\sum_{k=1}^{K} r_{k}\left(\mathbf{A}_{k}^{r}-\lambda_{k} \mathbf{B}_{k}^{r}\right)}_{\mathbf{C}(\mathbf{w})} \mathbf{w}
$$

where $\lambda=\left[\cdots, \lambda_{k}, \cdots\right]^{\mathrm{T}}, \forall k$. Equation (8) is similar to (3) where $r_{k}(\mathbf{w}), \lambda_{k}(\mathbf{w})$ and $\mathbf{C}_{k}(\mathbf{w})$ are projected according to (6) and (7) during the iterative procedure that finds the closest root of (5). As given by the Almogy/Levin iteration, $\lambda_{k}(\mathbf{w})$ will always increase, thereby increasing (1). The proposed multichannel TEQ design algorithm is outlined in Fig. 1. 


\section{Simulation Results}

The simulation results show the performance of the proposed multichannel shortening MCSSNR method vs. joint MSSNR channel shortening method by Melsa, Younce, and Rohrs. The comparison was achieved by designing the best multichannel TEQ using the Joint MSSNR algorithm and the proposed MCSSNR method and evaluating the modified SSNR ratio for each of the transmission channels in the set. The final value reported for both methods is then the weighted sum of all the evaluated modified SSNR. The closer the final value is to $100 \%$, the more successful is the designed TEQ FIR in reducing the energy outside of the desired window for each of the transmission channels in the set. We also compare the bit rate/channel of both mentioned methods to the bit rate/channel achieved using single FIR TEQ method in [11], in which each channel under consideration has its own TEQ FIR.

We use the eight standard downstream CSA loops [1] convolved with transmit and receive filters as the test CIR. The transmit and receive filters are modelled as first-order high pass infinite impulse response (IIR) filters, which are designed to separate ADSL from the voice band $(0-4 \mathrm{kHz})$. (A double zero is located at $z=1$, while conjugate symmetric poles are located at $z=0.9799 \pm \mathrm{j} 0.0317$ ). All CIRs consist of 512 samples sampled at $2.208 \mathrm{MHz}$. We use the FFT size $N=512$ for standard downstream ADSL transmission with an SNR gap $\Gamma=11.8 \mathrm{~dB}$. All power values used in simulation are defined with respect to a $100 \Omega$ resistance. The signal power is $0.2475 \mathrm{~W}$ spread equally over all subchannels. AWGN power is equal to $-140 \mathrm{dBm} / \mathrm{Hz}$ over the bandwidth of $1.104 \mathrm{MHz}$ with the NEXT source being modelled as 49 ADSL disturbers. The NEXT power spectral density is defined in the ADSL standard [1]. The transmission delay $\Delta$ is chosen by using a line search with respect to the values of $p(\mathbf{w})$.

The bit rates/channel reported are averages of the bit rates for CSA loops 1-8. Channel bit rates are calculated using the estimated SNR and following the ADSL standard for $10^{-7}$ BER. DMT subchannels of interest ( 7 to 256 from [1]) are loaded with a randomly chosen two-bit constellation point at the transmitter. The symbols are first convolved with the CIR, then passed through the TEQ block designed by the proposed method, and finally passed through an FFT block. After the FFT block, the phase and magnitude distortion is removed, and a slicing operation is performed. The slicing operation compares the complex value received in a subchannel with the expected value so that SNR measurement can be derived from the power of the error averaged over 1000 symbols.
Table 1: A comparison of achieved values of the objective function $p(\mathbf{w})$ of the proposed MCSSNR method and the corresponding bit rate/channel, with the Joint MSSNR method. Parameters are $N=512, \nu=32$, input power of $\mathbf{0} .2472 \mathbf{W}$, AWGN power of $-140 \mathrm{dBm} / \mathrm{Hz}$, and NEXT from 49 ADSL disturbers. The bit rate/channel and objective function values reported are averages obtained from averaging results for all TEQ sizes from 2-32.

\begin{tabular}{|c|c|c|}
\hline Method & $p(\mathbf{w})$ & Avg. Bit Rate/ch \\
\hline \hline MCSSNR & $99.71 \%$ & $9.26 \mathrm{Mbps}$ \\
\hline Joint MSSNR & $99.18 \%$ & $9.07 \mathrm{Mbps}$ \\
\hline
\end{tabular}

Each of the $K$ channels contributed equally to the objective function, so $\beta_{k}=\frac{100 \%}{K}$ for all $k$. Another choice might favor a particular subset of channels if it is determined that they have characteristics that occur with higher frequency in the channel set.

Table 1 lists the values of the objective function and the corresponding bit rate/channel averaged over TEQ FIR sizes $M$ from 2 to 32 . The value of the objective function for the MCSSNR is larger than the value obtained using the solution from Joint MSSNR, and so is the achieved bit rate/channel.

Figure 2 shows bit rate/channel achieved by varying the number of samples of the TEQ FIR from 232 for Joint MSSNR and for MCSSNR initialization, and final TEQ for the CIRs of CSA loops 1-8. It also shows the bit rate/channel achieved using the TEQFB design in [11], in which each channel under consideration has its own per-tone TEQ filter bank. MCSSNR and Joint MSSNR for TEQ sizes 2 and 3 are within $100 \mathrm{kbps}$ of TEQFB results, however the performance of the joint methods falters for larger sizes. Although MCSSNR step (4) presents the highest objective measure achievable when every channel has its uniquely designed TEQ according to the MCSSNR measure, the shown bit rate/channel results for step (4) are often worse than that achieved by the multichannel TEQ obtained in step (14). Fig. 2 shows that a maximization of an SSNR-based measure does not directly correlate to the maximization of the bit rate as we might expect (as opposed to results in [11]).

Figure 3 shows the shortening of CSA loops 1 and 5 by using a multichannel TEQ designed using MCSSNR on a set of CSA loops 1-8. 


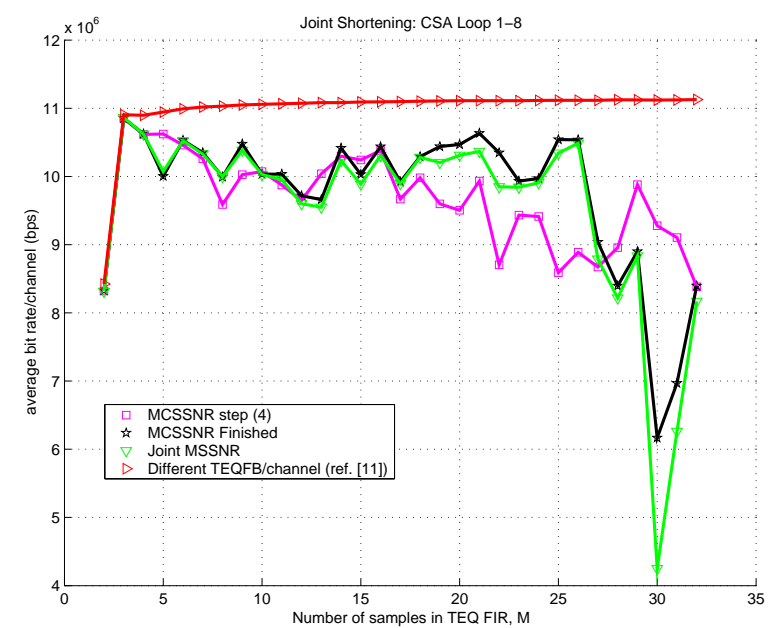

Figure 2: Data rates/channel averaged over 2-32 TEQ taps $(M)$ for the CIR containing CSA loops 18 with $N=512, \nu=32$, input power of $0.2472 \mathbf{W}$, AWGN power of $-140 \mathrm{dBm} / \mathrm{Hz}$, and NEXT modelled as 49 ADSL disturbers.

\section{Conclusion}

In this paper, we propose a new multichannel TEQ design method that will shorten multiple channels simultaneously. We arrive at the single TEQ by maximizing a new objective function that captures the contributions of different channels in a weighted sum of SSNR-based ratios.

\section{References}

[1] ANSI, "Network and customer installation interfaces: Asymmetric digital subscriber line (ADSL) metallic interface," in American National Standard for Telecomm., no. T1E1.413, 1998.

[2] ITU-T, "Asymmetrical digital subscriber line (ADSL) transceivers," in Int. Telecomm. Union, no. G.992.1, 1999.

[3] ITU-T, "Splitterless asymmetric digital subscriber line (ADSL) transceivers," in Int. Telecomm. Union, no. G.992.2, 1999.

[4] VDSLalliance, "SDMT VDSL draft standard proposal," Tech. Rep. T1E1.4/97-332, American National Standard for Telecomm., 1997.

[5] ETSI, "Digital video broadcasting (DVB); interaction channel for digital terrestrial television (DVBRCT)incorporating multiple access OFDM," Tech. Rep. EN 301958 DVB-RCT, ETSI, 2001.

[6] ETSI, "Broadband radio access networks (BRAN); HIPERLAN-2; physical layer," tech. rep., ETSI, 2000.

[7] N. Al-Dhahir and J. Cioffi, "Optimum finite length equalization for multicarrier transceivers," IEEE Trans. on Comm. vol. 44, pp. 56-64, Jan. 1996.

[8] G. Arslan, B. L. Evans, and S. Kiaei, "Equalization for discrete multitone transceivers to maximize bit rate," IEEE Trans. on Signal Proc., vol. 29, pp. 845-866, Dec. 2001.
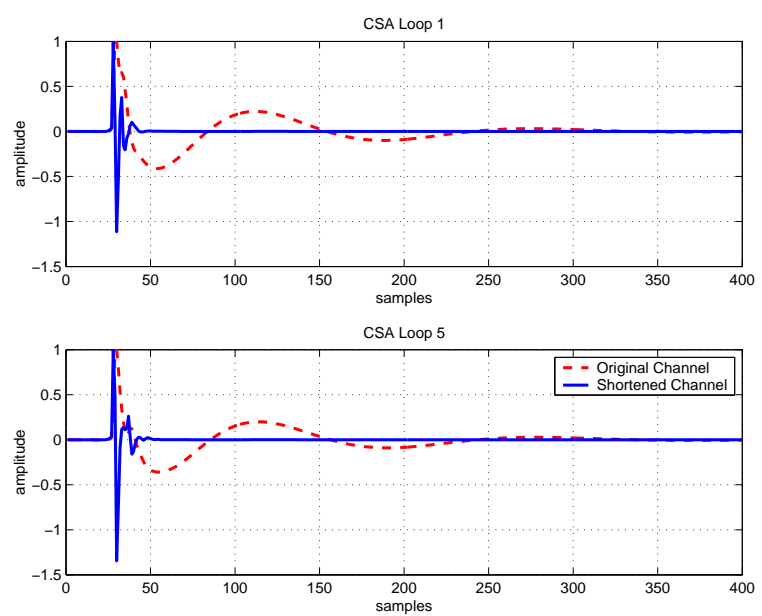

Figure 3: MCSSNR channel shortening of CSA loops 1-8. The figure shows a sample of results achieved for the CIR containing CSA loops 1 and $\mathbf{5}$ for $N=$ $512, \nu=32$, and $M=3$. Transmission delay $\Delta$ was found using a line search. The TEQ taps for $M=3$ are $\mathbf{w}=[0.4135,-0.8161,0.4037]^{\mathrm{T}}$.

[9] B. Farhang-Boroujeny and M. Ding, "An eigen-approach to design of near-optimum time domain equalizer for DMT tranceivers," in IEEE Int. Conf. on Comm., pp. 937-941, 1999.

[10] P. Melsa, R. Younce, and C. Rohrs, "Impulse response shortening for discrete multitone transceivers," IEEE Trans. on Comm., vol. 44, pp. 1662-1672, Dec. 1996.

[11] M. Milosevic, L. F. C. Pessoa, B. L. Evans, and R. Baldick, "DMT bit rate maximization with optimal time domain equalizer architecture," in Proc. IEEE Asilomar Conf. Signals, Systems, and Computers, Nov. 2002.

[12] J. S. Chow, J. M. Cioffi, and J. A. C. Bingham, "Equalizer training algorithms for multicarrier modulation systems," in Proc. IEEE Int. Conf. on Comm., pp. 761-765, May 1993.

[13] B. Farhang-Boroujeny and M. Ding, "Design methods for time-domain equalizers in DMT transceivers," IEEE Trans. on Comm., vol. 49, pp. 554-562, Mar. 2001.

[14] S. Schaible, "Fractional programming - a recent survey," Journal of Statistics and Management Systems, vol. 29, pp. 845-866, Mar. 2001.

[15] R. Freund and F. Jarre, "Solving the sum-of-ratios problem by an interior-point method," Tech. Rep. 3/99, Bell Labs, 1999.

[16] Y. Almogy and O. Levin, "A class of fractional programming problems," in Operations Research, vol. 19, pp. 57-67, 1971.

[17] J. Demmel, Applied Numerical Linear Algebra, pp. 173-176. SIAM, 1997.

[18] D. P. Bertsekas, Nonlinear Programming, pp. 54-75, 253. Athena Scientific, 1995.

[19] J. E. Falk and S. W. Palocsay, Optimizing the Sum of Linear Fractional Functions, pp. 221-258. Kluwer Academic Publishers, 1992. 